\title{
RESIDUALLY FINITE GROUPS
}

\section{BY WILHELM MAGNUS ${ }^{1}$}

1. Introduction. The definition of groups in terms of generators and defining relations became important when $\mathrm{H}$. Poincaré discovered the fundamental (or first homotopy) group of an arcwise connected topological space. In many cases, these fundamental groups can be defined easily in terms of generators and defining relations but not otherwise in a purely group theoretical manner (i.e. without reference to the underlying space).

We shall write

$$
\left\langle a_{\sigma} ; R_{\lambda}\right\rangle
$$

for a group $G$ with generators $a_{\sigma}$ and defining relations $R_{\lambda}=1$. Here $\sigma, \lambda$ are, respectively, elements of indexing sets $\Sigma, \Lambda$ where $\Sigma$ is nonempty, and the $R_{\lambda}$ (the "relators") are finite sequences or words in the $a_{\sigma}, a_{\sigma}^{-1}$. The unit element is denoted by 1 . If $\Lambda$ is empty, $G$ is called free, and the $a_{\sigma}$ are called a set of free generators. We shall call (1.1) a presentation of $G$ and shall talk respectively of finitely generated, finitely related and finitely presented groups whenever $\Sigma$ or $\Lambda$ or both are finite sets.

It is rather obvious that any sets of symbols $a_{\sigma}, a_{\sigma}^{-1}$ and words $R_{\lambda}$ in these symbols define a group. However, it turns out to be extremely difficult to develop methods which allow one to extract information about groups given by a presentation (1.1) in a purely algebraic manner. The fundamental problems arising here were formulated and investigated by Dehn [17]. The first of these, the word problem, is the question: Which words in the generators of a group $G$ represent the unit element? It became famous when Novikov [47], Boone [13] and Britton [15] exhibited finitely presented groups in which there is no general and effective procedure for determining whether a word in the given generators represents the unit element as a consequence of the given defining relations.

Investigating certain finitely presented groups arising from topology, Dehn [17], [18] found the available algebraic methods inadequate for this purpose and introduced geometric (including topologi-

An address delivered before the Baltimore meeting of the Society on October 26, 1968 by invitation of the Committee to Select Hour Speakers for Eastern Sectional Meetings; received by the editors November 20, 1968.

1 Preparation of this article was supported in part by the National Science Foundation. 
cal) methods to solve group-theoretical problems. Even now, twothirds of a century and many hundred research papers later, there are some problems concerning fundamental groups of topological spaces which can be solved easily by topological methods but not at all algebraically. A simple example is provided by the fundamental groups $\Phi_{o}$ of two-dimensional, closed, orientable manifolds of genus $g \geqq 1$. $\Phi_{g}$ has a presentation

$$
\left\langle a_{1}, a_{2}, \cdots, a_{2 g-1} a_{2 \sigma} ; \quad \prod_{\nu=1}^{0} a_{\nu}^{-1} a_{\nu+\theta}^{-1} a_{\nu} a_{\nu+\theta}\right\rangle
$$

as a one-relator group. All subgroups of $\Phi_{g}$ which are of finite index in $\Phi_{g}$ are again groups $\Phi_{h}$ where $h \geqq g$. But for $g>1$, this has not been proved algebraically, although it follows topologically from basic theorems about the classification of closed two-dimensional manifolds and about covering spaces.

The oldest and simplest way of obtaining at least partial information about a group $G$ presented in the form (1.1) is to abelianize it which means studying the quotient group $G / G^{\prime}$ of the commutator subgroup (also called the first derived group) of $G$. Generalizing this approach, one may try to find, for a given $G$, quotient groups which are "well known" for instance in the sense that they have a solvable word problem, and sufficiently numerous in the sense that knowing them provides a solution of the word problem in $G$. This situation will arise if, for every element $g \in G, g \neq 1$, there exists a finite homomorphic image $G^{*}$ of $G$ such that $g^{*} \neq 1$, where $g^{*}$ is the map of $g$ in $G^{*}$. Such a group $G$ is said to be residually finite. Of course, not all groups have this property. For instance, G. Higman [28] gave an example of an infinite group with four generators and four defining relations, the only finite quotient group of which is the trivial one. However, we can describe classes of groups which are residually finite and which are of interest either because of other group theoretical properties or because they occur in problems of geometry.

2. Residual properties. Let $C$ be a nonempty class of groups (which may, however, consist of a single group only). Following P. Hall, we shall say that a given group $G$ is residually $C$ if, for any element $g \neq 1$ in $G$ there exists a quotient group $G^{*}(g)$ belonging to $C$ such that the map $g^{*} \in G^{*}(g)$ of $g$ is not the unit element of $G^{*}(g)$. Equivalently, we can say that the intersection of all those normal subgroups $N$ of $G$ for which $G / N$ belongs to $C$ is the unit element of $G$. If the groups of the class $C$ are characterized by a particular property $\pi$ (e.g. by the property $\pi_{f}$ of being finite) we shall also say that $G$ is residually $\pi$. 
If $G$ is residually $C$ and if $C^{\prime}$ is a class of groups such that each group in $C$ is residually $C^{\prime}$, then $G$ is also residually $C^{\prime}$. To give examples, let $F_{n}$ denote the free group on $n$ free generators. Then, for $n \geqq 2, F_{n}$ is residually $F_{2}$ (see [48]) and $F_{2}$ is residually finite [24], therefore $F_{n}$ is residually finite for all $n$ since $n=1$ is a trivial case. Or consider the groups $\Phi_{g}$ defined by (1.2). It can be shown [20] that, for $g \geqq 2, \Phi_{g}$ is residually $F_{2}$ and therefore residually finite. Because of Property II of residually finite groups (as formulated below), this result has topological implications [31].

For any finitely generated residually finite group $G$ the following theorems have been proved:

I. G has a solvable word problem (A. Mostowski [43], McKinsey [40]).

II. $G$ is hopfian, that is every homomorphic mapping of $G$ onto itself is an automorphism (Malcev [39]).

III. The automorphism group of $G$ is residually finite (G. Baumslag. For a proof see p. 414 in [37]). This proves that the automorphism groups of $F_{n}, \Phi_{g}$, and also Artin's braid groups [1] are residually finite.

IV. If $G$ is infinite, it is a totally disconnected topological group with a nontrivial topology in which the normal subgroups of finite index form $a$ basis for the open sets containing the unit element. The completion $\bar{G}$ of $G$ under this topology is the inverse (also called "projective") limit of a sequence of finite quotient groups of $G$ (i.e. $G$ is a profinite group as defined by Serre [51]). For details and results see Marshall Hall, Jr. [24].

Obviously, all subgroups of a residually finite group $G$ are also residually finite. However, this need not be true for all quotient groups of $G$. For instance, $F_{2}$ is residually finite but its quotient group $B$ defined by

$$
\left\langle a, b ; a^{-1} b^{2} a b^{-3}\right\rangle
$$

is not. The proof (due to G. Baumslag) is so simple that it can be given in a few lines. Let $B^{*}$ be a finite homomorphic map of $B$ and let $\alpha, \beta$ be the maps of $a, b$. We have from (2.1) that

$$
a^{-n} b^{2^{n}} a^{n}=b^{3^{n}} \text {. }
$$

Therefore, if $\alpha$ is of order $n$, the order of $\beta$ divides

$$
k=3^{n}-2^{n} \text {. }
$$

Since $k$ is coprime with 2 and $3, \beta$ is a power of $\beta^{2}$ and the map $\beta_{1}$ of $b_{1}=a^{-1} b a$ in $B^{*}$ is a power of $\beta_{1}^{2}$ which in turn is $\beta^{3}$. Therefore the map 
of

$$
c=b_{1}^{-1} b^{-1} b_{1} b
$$

in any finite quotient group $B^{*}$ of $B$ is the unit element. However it can be shown by standard methods [35] that $c \neq 1$ in $B$, and therefore $B$ is not residually finite. (It can even be shown [12] that $B$ is nonhopfian.)

K. Gruenberg [22] introduced a class of properties of groups which have essential features in common with the property $\pi_{f}$ (of being finite). They are called root properties and are defined as follows:

A property $\rho$ is called a root property of a group $G$ if :

(i) All subgroups of $G$ also have property $\rho$.

(ii) The direct product of any two groups with property $\rho$ again has property $\rho$.

(iii) Given any three groups $G_{0} \supset G_{1} \supset G_{2}$, each normal in its predecessor and such that $G_{0} / G_{1}$ and $G_{1} / G_{2}$ have property $\rho$, there exists a subgroup $N$ in $G_{2}$ which is normal in $G_{0}$ such that $G_{0} / N$ also has property $\rho$.

Important root properties are: Being finite, being solvable, and the property denoted by $\pi(p)$ which means "being a group of order a finite power of the prime number $p$. " However, nilpotence is not a root property since it fails to satisfy (iii). Gruenberg [22] proved:

If $\rho$ is a root property, then every free product of residually $\rho$-groups is itself residually $\rho$ if and only if every free group is residually $\rho$.

Since free groups are residually $\pi(p)$ for all primes $p$ [24] and, therefore, residually finite and residually solvable, free products of respectively residually $\pi(p)$, residually finite, residually solvable groups have the same residual properties. Of course, residually $\pi(p)$ implies residual finiteness, but solvability (let alone residual solvability) does not, not even if we restrict ourselves to solvable groups $S$ with maximum condition (=ascending chain condition) for normal subgroups. ( $S$ will then be finitely generated.) A counterexample constructed by Gruenberg [22] is based on the following theorem [22]:

The (restricted) wreath product of the group $G$ by the group $U$ (where $U$ is in its regular representation as a permutation group) is residually finite if and only if both $G$ and $U$ are residually finite and either $U$ is finite or $G$ is abelian.

The counterexample mentioned above will be obtained by choosing for $U$ an infinite cyclic group and for $G$ the nonabelian group of order six. 
3. Residual finiteness. A large and important class of residually finite groups is described by the following theorem proved by A. Mal'cev [39].

Let $R$ be a commutative field and let $M$ be a finite set of $n$-by- $n$ matrices with elements in $R$ and with nonvanishing determinants. Then the matrices in $M$ generate a residually finite group.

Mal'cev's theorem proves, for instance, that Fuchsian groups are residually finite. (These are the finitely generated groups of fractional linear substitutions with real coefficients and determinant +1 in a complex variable $z$ which are discontinuous in the upper $z$-halfplane.) Some of them play an important role in the theory of complex variables (uniformization theorems) and in number theory. Their presentations in terms of generators and defining relations have been given by Fricke [21]. The fundamental groups $\Phi_{o}$ in (1.2) are special cases of Fuchsian groups. So are the groups resulting from the reflections of a noneuclidean triangle in its sides; they are defined by two generators $a, b$ and defining relations

$$
a^{\alpha}=b^{\beta}=(a b)^{\gamma}=1
$$

where $\alpha, \beta, \gamma$ are positive integers such that

$$
\frac{1}{\alpha}+\frac{1}{\beta}+\frac{1}{\gamma}<1 \text {. }
$$

They have been investigated repeatedly [19], [41], but their residual finiteness cannot yet be proved in all cases without using their geometric definition.

Naturally, free groups have an abundance of residual properties because of the basic property of $F_{n}$ that every group on $n$ generators is a quotient group of $F_{n}$. Free groups are residually nilpotent [36], that is, the groups of their lower central series intersect in the unit element. They are also [32], in the case of a rank $\geqq 2$, residually $T_{n}$, where $T_{n}$ is the single-relator group

$$
\left\langle a, b ; a^{n}\right\rangle
$$

and possibly residually $S$ for a large number of other single-relator groups $S$. For $n \geqq 2, F_{n}$ is not only residually $\pi(p)$ for every prime number $p$ [24], it is also residually $A$ and residually PSL $\left(2, p^{k}\right)$ for fixed $k$ and variable $p$, where $A$ denotes the class of alternating groups (or merely the class of alternating groups of degree $p$ ) and PSL $\left(2, p^{k}\right)$ denotes the class of groups of fractional linear substitutions with determinant +1 , in a single variable and over a field of $p^{k}$ elements [48], [32]. (Being residually $A$ also implies that in $F_{n}$ the subgroups 
of prime index intersect in the unit element [32].) Here, incidentally, the story ends. It is not even known whether $F_{2}$ is residually PSL $(3, p)$ where $p$ runs through all primes or residually PSL $\left(2,2^{k}\right)$ where $k$ runs through the positive integers.

The theorems stating residual finiteness for certain classes of groups $G$ use mostly one of two types of assumptions:

I. $G$ contains a finite sequence of subgroups $G_{\nu}, \nu=0,1, \cdots, n$, where $G_{0}=G, G_{n}=1$ and where $G_{v+1}$ is normal in $G_{\nu}$ such that $G_{\nu} / G_{\nu+1}$ belongs to a specified, rather restricted class of groups, being for instance a finitely generated group of a nontrivial variety. (For this concept see the expository article by B. H. Neumann [45] or the monograph by Hanna Neumann [46].)

II. $G$ is the generalized free product of two groups $G_{1}, G_{2}$ with an amalgamated subgroup $H$, where $G_{1}, G_{2}$ are residually finite or in specified classes of residually finite groups and where the restrictions on $H$ are rather severe. Nevertheless, the results using assumptions of this type appear to be very promising for the investigation of some groups arising in topology, particularly knot groups.

The first theorem using assumptions of type I is due to $\mathrm{K}$. Hirsch [30] and states:

Polycyclic groups are residually finite.

There are several equivalent definitions for a polycyclic group $G$. They are:

(i) There exists a finite sequence

$$
G=G_{0}, G_{1}, G_{2}, \cdots, G_{k}=1
$$

of subgroups $G_{v}$, each normal in the preceding one, such that $G_{v} / G_{v+1}$ is cyclic.

(ii) $G$ is solvable (i.e. the derived series $G^{\prime}, G^{\prime \prime}, \ldots$ terminates with the unit element after finitely many steps) and $G / G^{\prime}, G^{\prime} / G^{\prime \prime}, \ldots$ are all finitely generated.

(iii) $G$ is solvable and satisfies the maximum (=ascending chain) condition for subgroups.

Special cases of polycyclic groups are the finitely generated nilpotent groups (i.e. groups for which the lower central series terminates with the unit element after a finite number of steps). Gruenberg [22] showed that "residually nilpotent" and "residually of prime power order" are identical properties for finitely generated groups.

A strong generalization of Hirsch's theorem was proved by P. Hall [26]:

Every finitely generated group $G$ with an abelian normal subgroup $N$ and nilpotent quotient group $G / N$ is residually finite. 
P. Hall observes that groups of this type have the property that all of their quotient groups are also residually finite. They are, in his words, residually finite by general dispensation, whereas free groups share this property only by special grace. Earlier (Theorem 7 of [25]) P. Hall had shown that even finitely generated solvable groups may not be residually finite if one goes beyond metabelian groups $M$ (i.e. groups for which $M^{\prime \prime}=1$ ). He constructed a two-generator group $G$ the center $C$ of which is an arbitrary countable abelian group (which, obviously, need not be residually finite) such that $G / C$ is metabelian.

The sharpest results about residually finite solvable groups seem to be those of D. J. S. Robinson [49] who proved:

Let $A_{0}$ be the class of abelian groups in which the maximum number of generators for all finitely generated torsion free subgroups and for all finitely generated subgroups of order a power of $p$ (any prime number) is bounded. Let $S_{0}$ be the class of groups $G=G_{0}$ for which there exists a finite sequence of subgroups $G_{0}, G_{1}, \cdots, G_{n}=1$, each normal in the preceding one, such that $G_{\nu} / G_{\nu+1}$ belongs to $A_{0}$ for $\nu=0,1, \cdots, n-1$. Then a group in $S_{0}$ is residually finite and every maximal subgroup is of finite index if and only if the center of its Fitting subgroup (= the product of all normal nilpotent subgroups) is reduced.

Whereas even the finitely generated groups of most solvable varieties of groups are not always residually finite, the free groups of these varieties fare much better. K. Gruenberg [22] showed that any free polynilpotent group is residually $\pi(p)$ for all but a finite number of primes $p$ and remarks that a result of $P$. Hall even allows one to state this result for all $p$. Here a free polynilpotent group is defined as follows: Let $F$ be a free group, let $F_{i_{1}}$ be the $i_{1}$ th group of its lower central series (where $F=F_{1}$ ), and define recursively

$$
F_{i_{1}, i_{2}}, \cdots, i_{n},\left(i_{2}, i_{1}, \cdots, i_{n} \text { positive integers }\right)
$$

as the $i_{n}$ th group of the lower central series of

$$
F_{i_{1}, i_{2}, \cdots, i_{n-1}} \text {. }
$$

Then the quotient group of $F$ with respect to the group (3.1) is called free polynilpotent of class row,

$$
\left(i_{1}-1, i_{2}-1, \cdots, i_{n}-1\right) .
$$

A generalization of this result is the following: Let the finite group $G$ be presented in the form $F / R$, where $R$ is a normal subgroup of the free group $F$ which shall have a rank not less than 2 . Then Lihtman [34] showed (generalizing Gruenberg [23]): 


$$
F / R_{i_{1}, i_{2}}, \cdots, i_{\text {in }}
$$

is residually nilpotent if and only if the order of $G$ is a power of a prime.

G. Baumslag [10] showed: If $U$ and $V$ are varieties of groups, and if $U V=W$ denotes the variety of groups $G$ having a normal subgroup $N$ in $U$ such that $G / N$ belongs to $V$, then the free groups of $W$ are residually $F_{2}(W)$, where $F_{2}(W)$ is the free two-generator group of $W$.

For certain varietal products (generalized "verbal" products as introduced by S. Moran [42]) of groups in a variety $U$ with abelian groups, G. Baumslag [6] characterized those groups which are residually torsion free nilpotent.

We shall turn now to results based on assumptions of type II. Their significance is due to the fact that the generalized free products are the most versatile tool in the investigation of finitely presented groups. The solution of the word problem for single-relator groups [35], the extraction of roots of group elements [35], [44] and the construction of many fundamental groups, including the groups $\Phi_{\theta}$ in 2.1 (for $g>1$ ) and certain knot groups described below, all are applications of the theory of generalized free products. Another application is the construction of a nonhopfian (and therefore not residually finite) finitely presented group by G. Higman [27] which was the first one discovered. It is the group defined by

$$
\left\langle a, b, c ; a^{-1} c a c^{-2}, b^{-1} c b c^{-2}\right\rangle
$$

which is the generalized free product of the one-relator group

$$
\left\langle a, c ; a^{-1} c a c^{-2}\right\rangle, \quad\left\langle b, c ; b^{-1} c b c^{-2}\right\rangle
$$

with the cyclic subgroups generated by $c$ amalgamated. Although these one-relator groups are still residually finite, they have a rather complicated subgroup structure. However, even the generalized free product of two isomorphic two-generator groups which are nilpotent of class two and where the amalgamated subgroups are free abelian of rank two need not be hopfian (let alone residually finite), as was shown by G. Baumslag [3].

For free products (without amalgamations) of residually finite groups, Gruenberg's theorem on root properties establishes residual finiteness. What is known, in the line of positive results, about generalized free products is largely summarized in a paper [5] by G. Baumslag, who also reports on some unpublished work by G. Higman. Some of the results are:

Let $A, B$ be groups, and let $H$ and $K$ be, respectively, subgroups of 
$A$ and $B$ which are mapped isomorphically onto each other by an isomorphism $\theta$. Then the free product of $A$ and $B$ with amalgamation of $H$ and $K$ under $\Theta$ is residually finite if

$A$ and $B$ are finite or if

$A$ and $B$ are residually finite and $H$ is finite or if

$A$ and $B$ are finitely generated and nilpotent and if $H$ is cyclic or if $H$ as well as $K$ contains any element $x$ if it contains a power of $x$, or if $A$ and $B$ are polycyclic and $K$ is in the center of $B$, or if

$A$ and $B$ are polycyclic, $H$ is normal in $A$ and $K$ is normal in $B$.

If $A$ and $B$ are finitely generated and nilpotent, any generalized free product with amalgamated subgroups is still the extension of a free group by a residually finite group. The same is true if $A$ and $B$ are merely residually finite and if the isomorphism between $H$ and $K$ can be extended to an isomorphism between $A$ and $B$.

Other results by G. Baumslag [4], [11] state that a generalized free product of a free group $F$ and a free abelian group $A$ of countable rank is residually free (and thus residually finite) if the amalgamated subgroup is infinite cyclic, its own centralizer in $F$, and generated by a free generator of $A$. Also, a free group arising from $F$ by extraction of an $m$ th root is residually finite. This is a generalization of a result found by $\mathrm{B}$. Chandler [16] which in turn shows the residual finiteness of the groups $\phi_{g}$. The proofs depend on realizations of free groups as subgroups of the multiplicative group of rings; in Chandler's paper, the ring is a matrix representation of a ring introduced by Malcev, whereas Baumslag constructs new rings for his purposes. G. Baumslag also proved residual finiteness for certain one-relator groups which contain elements $\neq 1$ of finite order [9].

P. Stebe [52] used generalized free products to prove residual finiteness for a class of knot groups. The group property needed here will be called $\pi_{c}$; it is stronger than residual finiteness and is defined as follows: $G$ is $\pi_{c}$ if for any two elements $g_{1}, g_{2}$ in $G$ which are such that for all integers $m$

$$
g_{1} \neq g^{m},
$$

there exists a finite homomorphic image $G^{*}$ such that the maps $g_{1}^{*}, g_{2}^{*}$ of $g_{1}, g_{2}$ in $G^{*}$ also satisfy

$$
g_{1}^{*} \neq g_{2}^{* m} .
$$

(If we chose $g_{2}=1$, we have the condition for residual finiteness.) Now we have:

Let $A_{\eta}, \nu=1, \cdots, n$, be finitely generated, isomorphic groups 
which are $\pi_{c}$, and let $\Theta_{\nu}$ be the isomorphisms mapping $A_{1}$ onto $A_{\nu}$. Let $C_{1}$ be a cyclic subgroup of $A_{1}$ and let $C_{\nu}$ be its map under $\theta_{\nu}$. Let $G$ be the generalized free product of the $A_{\nu}$ with amalgamation of $C_{1}$ and $C_{\nu}$ (for $\nu=2, \cdots, n$ ) under the isomorphism $\Theta_{\nu}$. Then $G$ is $\pi_{c}$. (It should be noted that the isomorphisms between the amalgamated subgroups are extended to the factors of the generalized free product.)

As a consequence we have: Let $G$ be finitely generated and $\pi_{c}$. Let $G_{1}$ be defined by forming first the free product of $G$ with an infinite cyclic group generated by an element $x$ and then adding either one of the two relations

$$
x^{m}=g, x^{-1} g^{-1} x g=1 ; \quad g \in G .
$$

Then $G_{1}$ is also $\pi_{c}$. This implies that the knot groups defined by Brauner [14] are residually $\pi_{c}$. These knot groups form a rather large class of groups which are defined only recursively. They are of special interest since they are associated with the algebraic-type singularities of analytic functions of two complex variables.

As a last item to report, it should be mentioned that residually finite groups $G_{1}$ and $G_{2}$ need not be isomorphic if their sets of finite quotient groups coincide. An example (still unpublished) was found by Joan Landman-Dyer. In this example, all possible finite quotient groups of $G_{1}$ and $G_{2}$ are nilpotent. Earlier, G. Baumslag [8] had already shown that there exist groups $G$ which are residually nilpotent and not free but for which the quotient groups $G / G_{n}$ of the groups $G_{n}$ of the lower central series and also $G / G^{\prime \prime}$ coincide with those of a finitely generated free group $F$. This implies that the sets of all nilpotent and all metabelian quotient groups of $F$ and $G$ coincide.

Some of the references listed below are not referred to in the text but are relevant to the topics mentioned there.

\section{REFERENCES}

1. E. Artin, Theorie der Zoepfe, Abh. Math. Sem. Univ. Hamburg 4 (1925), 47-72.

2. B. Baumslag, Residually free groups, Proc. London Math. Soc. (3) 17 (1967), $402-418$.

3. G. Baumslag, A non-hopfian group, Bull. Amer. Math. Soc. 68 (1962), 196-198.

4. - On generalized free products, Math. Z. 78 (1962), 423-438.

5. - On the residual finiteness of generalized free products of nilpotent groups, Trans. Amer. Math. Soc. 106 (1963), 193-209.

6. - On the residual nilpotence of some varietal products, Trans. Amer. Math. Soc. 109 (1963), 357-365. 271-282.

7. - Residual nilpotence and relations in free groups, J. Algebra 2 (1965),

8. - Some groups that are just about free, Bull. Amer. Math. Soc. 73 (1967), 621-622. 
9. - Residually finite one-relator groups, Bull. Amer. Math. Soc. 73 (1967), 618-620.

10. - Some theorems on the free groups of certain product varieties, J. Combinatorial Theory 2 (1967), 77-99.

11. - On the residual nilpotence of certain one-relator groups, Comm. Pure Appl. Math. 21 (1968), 491-506.

12. G. Baumslag and D. Solitar, Some two-generator one-relator non-hopfian groups, Bull. Amer. Math. Soc. 68 (1962), 199-201.

13. W. W. Boone, Certain simple unsolvable problems of group theory, Indig. Math. 16 (1955), 231-237, 492-497; 17 (1955), 252-256; 19 (1957), 22-27, 227-232.

14. K. Brauner, Zur Geometrie der Funktionen zweier komplexer Veraenderlicher, Abh. Math. Sem. Univ. Hamburg 6 (1928), 1-55.

15. J. L. Britton, The word problem for groups, Proc. London Math. Soc. (3) 8 (1958), 493-506.

16. B. Chandler, The representation of a generalized free product in an associative ring, Comm. Pure Appl. Math. 21 (1968), 271-288.

17. M. Dehn, Ueberunendliche diskontinuierliche Gruppen, Math. Ann. 71 (1911), 116-144.

18. - Transformation der Kurven auf zweiseitigen Flaechen, Math. Ann. 72 (1912), 413-421.

19. Ralph H. Fox, On Fenchel's conjecture about F-groups, Math. Tidsskr. B. (1952), 61-65.

20. Karen Frederick, The Hopfian property for a class of fundamental groups, Comm. Pure Appl. Math. 16 (1963), 1-8.

21. R. Fricke and F. Klein, Vorlesungen über die Theorie der automorphen Funktionen. I, Teubner, Berlin, 1897, pp. 186-187.

22. K. W. Gruenberg, Residual properties of infinite soluble groups, Proc. London Math. Soc. (3) 7 (1957), 29-62.

23. - The residual nilpotence of certain presentations of finite groups, Arch. Math. 13 (1962), 408-417.

24. Marshall Hall, Jr., A topology for free groups and related groups, Ann. of Math.

(2) 52 (1950), 127-139.

25. P. Hall, Finiteness conditions for soluble groups, Proc. London Math. Soc. (3) 4 (1954), 419-436.

26. - On the finiteness of certain soluble groups, Proc. London Math. Soc. (3) 9 (1959), 595-622.

27. G. Higman, $A$ finitely related group with an isomorphic proper factor group, J. London Math. Soc. 26 (1951), 59-61.

28. - A Ainitely generated infinite simple group, J. London Math. Soc. 26 (1951), 61-64.

29. - A remark on finitely generated nilpotent groups, Proc. Amer. Math. Soc. 6 (1955), 284-285.

30. K. Hirsch, On infinite soluble groups. III, Proc. London Math. Soc. (2) 49 (1946), 184-194.

31. H. Hopf, Beiträge zur Klassifizierung der Flächenabbildungen, J. Reine Angew. Math. 165 (1931), 225-236.

32. R. Katz and W. Magnus, Residual properties of free groups, Comm. Pure Appl. Math. 22 (1969), 1-13.

33. A. Learner, Residual properties of polycyclic groups, Illinois J. Math. 8 (1964), 536-542. 

866.

34. A. I. Lihtman, On residually nilpotent groups, Sibirsk. Mat. Z. 6 (1965), 862-

35. W. Magnus, Das Identitaetsproblem fuer Gruppen mit einer definienenden Relation, Math. Ann. 106 (1932), 295-307.

36. - Beziehungen zwischen Gruppen und Idealen in einem speziellen Ring, Math. Ann. 111 (1935), 259-280.

37. W. Magnus, A. Karrass and D. Solitar, Combinatorial group theory, Wiley, New York, 1966.

38. W. Magnus and Ada Peluso, On knot groups, Comm. Pure Appl. Math. 20 (1967), 749-770.

39. A. Mal'cev, On isomorphic matrix representations of infinite groups, Mat. Sb. 8 (50) (1940), 405-422. (Russian)

40. J. C. C. McKinsey, The decision problem for some classes of sentences, J. Symbolic Logic 8 (1943), 61-76.

41. J. Mennicke, Eine Bemerkung ueber Fuchssche Gruppen, Invent. Math. 2 (1967), 301-305.

42. S. Moran, Associative operators in groups. I, Proc. London Math. Soc. 6 (1956), 581-596.

43. A. W. Mostowski, On the decidability of some problems in special classes of groups, Fund. Math. 59 (1966), 123-135.

44. B. H. Neumann, Adjunction of elements to groups, J. London Math. Soc. 18 (1943), 4-11.

45. —, Varieties of groups, Bull. Amer. Math. Soc. 73 (1967), 603-613.

46. H. Neumann, Varieties of groups, Springer-Verlag, Berlin, 1967.

47. P. S. Novikov, On the algorithmic insolvability of the word problem in group theory, Trudy Mat. Inst. Steklov. 44 (1955); English transl., Amer. Math. Soc. Transl. (2) 9 (1958), 1-122.

48. A. Peluso, A residual property of free groups, Comm. Pure Appl. Math. 19 (1967), 435-437.

49. D. J. S. Robinson, Residual properties of some classes of infinite soluble groups, Proc. London Math. Soc. (3) 18 (1968), 495-520.

50. K. Seksenbaev, On the theory of polycyclic groups, Algebra i Logika Sem. 4 (1965), no. 3, 79-83. (Russian)

51. Jean-Pierre Serre, Cohomologie Galoisienne, Lecture Notes in Mathematics, Springer-Verlag, Berlin, 1964.

52. P. Stebe, Residual finiteness of a class of knot groups, Comm. Pure Appl. Math. 21 (1968), 563-583.

53. A. Steinberg, On free nilpotent quotient groups, Math. Z. 85 (1964), 185-196. 